\title{
Branded content versus product placement. Visibilidad, recuerdo y percepción del consumidor
}

\author{
María Josefa Formoso Barro ${ }^{1}$; Antonio Sanjuán Pérez ${ }^{2}$; Sandra Martínez Costa ${ }^{3}$
}

Resumen. El product placement ha tenido siempre una alta eficacia, a pesar de ser una de las modalidades publicitarias más cuestionadas por resultar invasiva. El branded content sin embargo, ha contado siempre con mejores valoraciones entre los profesionales de la publicidad, pero queda por conseguir una correcta medición de su eficacia.

El presente artículo muestra los resultados de una investigación experimental de tipo comparativo entre las fórmulas publicitarias del product placement y el branded content de un mismo anunciante. La finalidad es la de identificar cuál de los dos métodos resulta más eficiente para la visibilidad y el recuerdo del producto anunciado, así como para la mejora de la percepción emotiva que el espectador tiene de la marca.

Para ello se ha realizado un estudio cualitativo con eye tracking y varios cuestionarios de preguntas abiertas y cerradas a dos grupos de usuarios diferentes, expuestos cada uno de ellos a una de las dos modalidades publicitarias. Los resultados del análisis indican que los valores comparativos de visibilidad y recuerdo difieren de los relativos a la percepción emotiva y subjetiva del espectador según el tipo de publicidad utilizada por la marca. Así, mientras el branded content ayuda a mejorar la visibilidad y recuerdo de la marca, los cambios más significativos sobre la percepción emotiva del producto se dan entre los consumidores que la han visto bajo la modalidad del product placement.

Palabras clave: Branded content; percepción; product placement; eficacia; recuerdo; visibilidad.

\section{[en] Branded content versus product placement. Visibility, perception and consumer's brand recall}

Abstract. Product placement has been long efficiency, despite being one of the most controversial advertising modalities for its invasive methods. However, the branded content has always been appreciated among advertising professionals, but it remains to get a correct measurement of its effectiveness.

This article is a result of an experimental research comparing advertising formulas of product placement and branded content from the same advertiser, in order to identify which one of both methods is more efficient for product visibility, brand recall and emotional perception improvement.

1 Doctora en Ciencias de la Información. Universidad Complutense de Madrid. Profesora Contratada Interina de Sustitución en la Universidade da Coruña.

m.formoso@udc.es

2 Doctor en Ciencias de la Información. Universidad Complutense de Madrid. Profesor Titular en la Universidade da Coruña.

sanjuan@udc.es

3 Doctora en Publicidad y Relaciones Públicas. Universidade de Vigo. Profesora Contratada Doctor en la Universidade da Coruña. smartinez@udc.es 
To do that, a qualitative study in two different user groups was made with eye tracking and some open and closed questions. Each group was exposed to one of the advertising methods. The analysis results indicate that the values of visibility and brand recall differ from those on the emotional and subjective perception depending on the advertising method used.

Keywords: Branded content; perception; product placement; efficiency; brand recall; visibility.

Sumario. 1. Introducción. 2. Objetivos de la investigación. 3. Metodología y herramientas. 4. Hipótesis de partida. 5. Resultados de la investigación. 6. Conclusiones. 7. Referencias bibliográficas.

Cómo citar: Assier-Andrieu, L. (2015) Dificultad y necesidad de la antropología del derecho, en Revista de Antropología Social 24, 35-52.

\section{Introducción}

La proliferación de medios y contenidos digitales, tanto para TDT como para otros nuevos soportes, así como el incremento en la capacidad de producción publicitaria y el abaratamiento de costes han hecho que las fórmulas y estrategias de las marcas y anunciantes se hayan diversificado, buscando impactar a un público cada vez más disperso, y también más exigente, segmentado y especializado.

El incremento del uso de dispositivos portátiles y de las conexiones wifi ha ayudado también al aumento de ese consumo disgregado (Rosique, G. 2010) ya que muchos usuarios navegan por contenidos audiovisuales en la web o en el móvil en lugar de hacerlo a través del televisor, de una forma más convencional, al tiempo que complementan lo que ven en una de las pantallas con información adicional en la otra. Y por otra parte se ha multiplicado la autogeneración de contenidos audiovisuales. El descenso en el precio de los productos tecnológicos, la mejora de la calidad de vídeo, el acceso y la popularización de las herramientas de edición y la multiplicación de plataformas broadcasting son factores por los que el usuario es cada vez más creador y difusor de contenidos de ocio, diversificando la oferta y convirtiéndose también en un consumidor más exigente pero a la vez menos fiel.

Esa tendencia ha sido a su vez seguida de forma masiva por las marcas, que ven por primera vez que la capacidad de producción y distribución de contenido publicitario está en sus manos, en lugar de en las grandes centrales de medios o en las cadenas de televisión. El contenido publicitario se ha multiplicado en internet, y es frecuente ver que las marcas cuentan con estrategias que buscan la participación de su público objetivo, con varios vídeos promocionales o incluso cortos de ficción, juegos, aplicaciones para móviles, participación en concursos, vídeos interactivos, etc. La finalidad es la de darse a conocer entreteniendo, emocionando o educando al público en algún modo. Si el contenido resulta interesante, el usuario será el que lo distribuya, lo que contribuye al éxito publicitario.

Pero al mismo tiempo los anunciantes siguen apostando por la publicidad en televisión, ya que es la forma más rápida de llegar al público masivo y dar a conocer el producto. $\mathrm{Y}$ en ese contexto es en el que el product placement sigue siendo una de las técnicas publicitarias de mayor eficacia (Verhellen, Y., Dens, N. \& De Pelsmacker, P. 2014). La inserción de marcas en productos audiovisuales de ficción o de entretenimiento (videojuegos, además de series y películas) esquiva el zapping. El producto anunciado está presente cada vez que el consumidor ve la película o serie en que fue insertado, independientemente del momento o del soporte en que acceda a él. Por eso mismo, su pervivencia es mucho mayor que la de un spot, el 
coste por impacto más bajo y si está correctamente utilizado, las ventajas narrativas que aporta al producto de ficción son muy altas (Baños, M. \& Rodríguez, T., 2004). Resulta una técnica publicitaria muy útil para posicionar productos en la mente del consumidor, ya que recoge la imagen de marca de la propia historia de ficción en la que es insertada y la imagen de los actores, y al mismo tiempo da realismo a los guiones. Su visibilidad y recuerdo suelen ser también muy altos entre el espectador (Del Pino, C. \& Olivares, F., 2006) aunque en muchas ocasiones sea por abuso o mal uso de las ubicaciones en las que se colocó la marca.

El product placement también está considerado por muchos como intrusivo y en ocasiones se ha forzado su presencia hasta el límite de saturar al público. Su uso indiscriminado resulta perjudicial ya que provoca rechazo y el localismo de los productos exhibidos hace que las marcas no sean reconocidas a nivel internacional por los consumidores, lo que convierte en ineficaz el emplazamiento. También ha sido cuestionado porque en muchas ocasiones ha escapado a la legalidad, especialmente en el caso de productos como el tabaco y el alcohol.

El product placement es definido como «la exhibición o mención de un producto, marca o logotipo reconocible, mostrada o utilizada de forma natural o sutil, previo pago por la empresa o instituciones responsables de la marca, con fines promocionales y/o comerciales» (Goupta, P. \& Gould, S. 19974). Sin embargo, se habla de diversas clasificaciones de product placement que pueden llegar a provocar confusión terminológica con el branded content.

La definición de brand placement (Karrh, J. A. 1998) ${ }^{5}$, por ejemplo, implica un paso que «va más allá de la mera inserción de marcas y productos a modo de attrezzo en un contenido audiovisual. » Del Pino y Olivares ${ }^{6}$ (2006) lo definen también como «toda presencia o referencia audiovisual intencional a una marca (de producto, empresa, famoso o mancomunada), claramente identificable, lograda mediante una gestión y negociación con la productora, integrada en el contexto espacial y/o narrativo del género de la ficción cinematográfica y televisiva» A pesar de la globalidad de esta definición, los autores hacen referencia al brand placement como una técnica integrada en la ficción que va más allá de la simple ubicación de marcas (product placement), dado que se trata de menciones en los guiones o del uso o consumo de los productos por parte de los personajes de la serie o película en que se inserta la marca. Se trata pues de emplazamientos activos de producto, en los que la marca tiene una presencia más relevante en la acción (Méndiz, A. 2000), pero no hay creación de contenidos propios, sino en la integración de la publicidad en productos de ficción que son ajenos a la marca.

Así, el branded content es definido como

Aquellas acciones en las que se busca integrar los valores de una marca en un contenido que puede ser de entretenimiento, información o educación ya sea a

4 Gupta, P. \& Gould, S. (1997) Consumers perceptions of the ethics and acceptability of product placement in movies: product category and individual differences, en Journal of Current Issues and Research Advertising 19 (1), 37-50.

5 Karrh, J. A. (1998) Brand Placement: A Review, en Journal of Current Issues \& Research in Advertising 20:2, 31-49. DOI: 10.1080/10641734.1998.10505081

6 Del Pino \& Olivares (2006) Brand placement y advertainment: integración y fusión entre la ficción audiovisual y las marcas, en Zer 22, 341-467. 
través del product placement o emplazamiento de producto, de los patrocinios, de las pantallas compartidas o de modalidades como el advertainment y el licensing (Aguado, 2008) ${ }^{7}$.

Es por lo tanto «una mezcla indiscernible entre entretenimiento y publicidad (o, si se prefiere, de una puesta del entretenimiento cinematográfico al servicio de la publicidad comercial)» (Caro, A. 2013) .

La idea es la de que el mensaje publicitario ha de integrarse todo lo posible en el contenido que el espectador vería de manera habitual, sin interrumpir sus momentos de ocio y formando parte de una estrategia publicitaria "pull" en lugar de "push", como sería en el caso del product placement. Sin embargo, la distinción entre las dos modalidades publicitarias se encuentra en la autoría del contenido que se convierte en "soporte publicitario". Se trata fundamentalmente de la producción e incluso distribución de contenidos de la marca cuya finalidad va más allá de lo meramente publicitario, lo que implica un cambio de relación en la comunicación entre esta y su target.

La producción de contenidos de ficción o de puro ocio para consumidores es una tendencia al alza entre los anunciantes y nace precisamente del abaratamiento y la mejora de calidad de los medios técnicos audiovisuales y del incremento en la capacidad de distribución de los mismos vía internet. La hibridación de géneros audiovisuales, favorecido en parte por la demanda de contenidos de bajo coste, provoca que cada vez sean más los anunciantes los que generan y venden a las cadenas televisivas productos de ocio, educativos o de ficción que están relacionados de forma directa o indirecta con sus marcas y productos. Se trata de una publicidad más emocional, menos basada en la exposición racional de las ventajas del producto y que, sobre todo, huye de la mención y la publicitación directa y masiva de la marca (Aguado, G. G. 2008). Y por lo tanto cuenta con una eficacia mucho menos medible y cuantificable.

En los últimos años destacan en España ejemplos como los de las campañas de Mercadona, Fanta o Nespresso para Atresmedia; o los de Coca Cola para Mediaset entre otros. Son contenidos que van más allá de lo publicitario y entran en lo divulgativo o en el mundo del ocio del consumidor. La marca está presente, pero de forma secundaria. Es mencionada, aparece fugazmente u ocupa una buena parte del programa, pero de cualquier forma lo fundamental es el producto audiovisual y lo secundario es la marca. Generalmente, el contenedor publicitario hace referencia de alguna manera a los valores intrínsecos de la marca. Por ello el anunciante ha de producir o co-producir el contenido e intervenir en el proceso de creación de la idea y los guiones en la medida de lo posible. El contenido ha de ser interesante para que su difusión sea exitosa, por lo que muchas veces se incorporan al mismo herramientas de participación de la audiencia que incrementan la implicación con el producto y ayudan a difundirlo. Los objetivos de este tipo de campañas deben ser los de entretener a los usuarios, desarrollar comunicaciones comerciales al servicio

7 Aguado, G. G. (2008) Branded content más allá del product placement en la televisión digital: Advertainment y licensing, en Revista del CES Felipe II, (8), 5. http://www.cesfelipesegundo.com/revista/articulos2008/ Art\%EDculo\%20Guadalupe\%20Aguado_corregido.pdf Web visitada en julio 2016.

8 Caro, A. (2013) Del spot al branded content: lo audiovisual al servicio de las marcas, en Conferencia magistral. Congreso Internacional de Comunicación Facetas 4. Universidad Autónoma de Baja California. Ensenada, 5.11.2013. En: http://www.academia.edu/5035067/2013_-_Del_spot_al_branded_content._Lo_audiovisual_ al_servicio_de_las_marcas. Web visitada en julio 2016. 
de los objetivos de la marca e implicar y comprometer al usuario con el producto o servicio.

Los anunciantes buscan entretener, emocionar, divertir o sorprender al espectador a través del branded content generando así un vínculo emocional con él. Dado que la idea es la de transmitir valores al consumidor esto es imprescindible. Esa transmisión de valores y conceptos es también uno de los principales objetivos del product placement. Los contenidos van asociados en la pantalla a guiones o personajes de vidas apasionantes, escenas de acción, elegancia o modernidad, entre otros aspectos. La marca se apropia de estas propiedades pero su implicación con el producto audiovisual es mucho menor con esta modalidad publicitaria que en el caso del branded content. En muchos casos la presencia de la marca no va mucho más allá de la mera ubicación en los decorados o como mucho, algunos guiones se adaptan al contenido para que los actores la mencionen o hablen de ella.

En cualquier caso, las dos modalidades publicitarias comparten la intención de no interrumpir el momento de ocio del espectador, sino formar parte del mismo al integrarse en el contenido de ficción. Pero existe una clara diferencia entre ellas: el product placement no es contenido buscado por el usuario, mientras que el branded content sí lo es.

Por otro lado, cabe destacar el hecho de que ambas son modalidades publicitarias más emocionales que racionales. Eso dificulta la medición de su eficacia, entendida como aquella que determina los objetivos y el cumplimiento de los mismos que tiene o ha de tener cada campaña o anuncio (Pergelova, A., Prior, D. \& Rialp, J. 2010). Para las dos modalidades estudiadas buena parte de su eficacia depende de factores como la predisposición del individuo en cada momento puntual, las diferencias emotivas entre consumidores, la intención de compra, la percepción de la marca, el recuerdo sobre el mensaje y otros elementos condicionados en gran medida por los valores y creencias previas del público, siempre desde un punto de vista personal e individual (Hollebeek, L. 2011). Además, los valores transmitidos por la marca son intrínsecos $\mathrm{y}$, por lo tanto, difíciles de cuantificar porque muchas veces son valores emocionales (López, B. 2007). Buscar herramientas que ayuden a entender la eficacia de estas técnicas publicitarias es por lo tanto fundamental, pero han de tenerse en cuenta los factores que influyen en la imagen funcional, la imagen afectiva y la reputación de una marca (Martínez, E. Montaner, T., Pina, J. M. 2005). La participación y difusión del contenido publicitario por parte del espectador es una de las formas de medir el éxito de un contenido publicitario. Las redes sociales aportan en ese sentido formas de medición cuantificables, pero que no indican necesariamente la notoriedad y relevancia que la marca tiene entre su público objetivo o el interés que ha generado en él. Mucho de ese contenido puede no ser fácil de compartir, y en ocasiones se utilizan los medios de comunicación tradicionales (especialmente cadenas de televisión) como vía de difusión, con lo que la participación del público no indica el alcance real que ha tenido el producto publicitario. Además, debe tenerse en cuenta el concepto de engagement, asociado a técnicas publicitarias como el branded content, y que busca no solo la asociación de la marca a una serie de valores sociales o culturales, sino también un posible cambio de comportamiento en el consumidor (Gambetti, R. \& Graffigna, G. 2010). Al margen queda ya la medición del éxito del product placement, que se limita casi en exclusiva a los estudios relativos a su recuerdo y aceptación entre el target, pero que cuenta con escasas herramientas de medición sobre la percepción emocional que tiene el espectador tras la exposición a esta modalidad publicitaria (Del Pino, C. \& Reinares, R. 2013). 


\section{Objetivos de la investigación}

El objetivo de este estudio es el de determinar si el branded content resulta más o menos eficiente que el product placement en términos de visibilidad, de percepción por parte del consumidor y de recuerdo de la marca anunciada. La selección de estas dos modalidades publicitarias en prejuicio de otras, viene determinada por el hecho de que ambas son muy parecidas en lo formal, pero opuestas en cuanto a la estrategia publicitaria (estrategias de pull o de push). Dado que existe una mejor predisposición del consumidor a la publicidad buscada por él mismo ( $p u l l$ ) que hacia la impuesta por la marca (push), es de suponer que los productos que utilizan el product placement como soporte publicitario obtendrán peores resultados sobre percepción emocional de marca. Sin embargo, se trata de una modalidad donde la visibilidad del producto es muy alta (Fernández, J. M., Reyes, I., Rocatagliata, E., Ruiz, E., Vargas, P. \& Pérez, J. P. 2012) y por lo tanto puede resultar más eficiente que el branded content que en muchas ocasiones pasa desapercibido a ojos del espectador o no alcanza el nivel de recuerdo deseado.

Por ello, para este trabajo de investigación se llevó a cabo un análisis comparativo de la presencia de la marca gallega de cervezas Estrella Galicia, utilizando la presencia de las dos modalidades publicitarias que son objeto de estudio en varios programas televisivos. Estrella Galicia es el producto de referencia de Hijos de Rivera S.A., empresa cervecera nacida en 1906 en A Coruña. En 2014 contó con una facturación de 280 millones de euros, en buena parte gracias a sus estrategias de promoción en medios de comunicación y redes sociales, de patrocinio de eventos y de mecenazgo, y a la expansión internacional que han llevado a cabo en los últimos años. Su actividad publicitaria no solo se centra en los spots para televisión, sino que también participa activamente en la ficción nacional e incluso internacional (mediante técnicas de product placement virtual, por ejemplo) y en contenidos de entretenimiento. Su estrategia en redes sociales le ha llevado a tener más de 206.000 seguidores en Facebook y más de 58.000 en Twitter?.

En la modalidad de product placement de la marca que fue analizada para este estudio el producto se encontraba ubicado en el escenario de una serie de ficción (Padre Casares, emitida desde 2008 en Televisión de Galicia), de forma pasiva la mayor parte del tiempo, aunque en algún momento puntual lo consumía uno de los actores secundarios. Este tipo de emplazamiento es habitual en la marca, aunque ha destacado por ubicaciones más impactantes y activas, como las de las campanadas en las cadenas de Mediaset y Atresmedia en el Fin de Año de 2015.

En el caso del branded content, la marca se insertaba como parte de una tarea que debían realizar los participantes del programa de entretenimiento El Hormiguero, emitido en Antena 3.

\section{Metodología y herramientas}

En general, las investigaciones sobre eficacia publicitaria de una marca anunciante se centran en los estudios sobre el recuerdo (Weller, R., Sibley, S. \& Neuhaus, C. 2015), la actitud del consumidor hacia la publicidad o hacia la marca anunciada (Gould, S.,

Última revisión en julio 2016. 
Gupta, P. \& Grabner-Kräuter, S. 2000); y el reconocimiento (Balasubramanian, S. K., Karrh, J. A. \& Patwardhan, H. 2006) y la visibilidad de la misma (Williams, K., Petrosky, A., Hernandez, E. \& Page, R. 2011).

Estos factores suelen ser utilizados como valores de medición independientemente de la modalidad que el anunciante haya utilizado para llegar a su público objetivo y por ello para este estudio se tuvieron en cuenta como medio de comparación de la eficacia para el product placement y el branded content analizados.

Para medir la eficacia publicitaria se propone un estudio con eye tracking realizado a una muestra de conveniencia o por oportunidad de 30 usuarios, con un perfil de edad de entre los 30 y los 45 años (17 mujeres y 13 hombres), dada su similitud en edad al target de la marca que es objeto de estudio. Para que no existiese comparación o resultase afectada la variable de recuerdo, se utilizaron dos grupos diferentes de quince personas, y cada uno de ellos fue expuesto a una modalidad publicitaria diferente. Puesto que autores como Regueira (2012) indican que el consumo habitual de la marca anunciada hace que la eficacia del branded content resulte mayor, se procuró que los 30 participantes del estudio fuesen consumidores de Estrella Galicia, de tal forma que no hubiese diferencias entre compradores y no compradores del producto. Este hecho implicaba por lo tanto que los consumidores contaban ya con una valoración y una predisposición sobre la marca que resultaba inevitable por tratarse de un producto de larga trayectoria en el mercado. Dicha predisposición se midió antes del comienzo de la exposición publicitaria, a través de preguntas de respuesta abierta sobre las marcas de cerveza consumidas habitualmente por los participantes del estudio, la frecuencia y el grado de sentimiento, con el fin de poder hacer una comparativa posterior y medir así la evolución de la percepción en el consumidor antes y después de la exposición al contenido publicitario.

El visionado de los contenidos se hizo con eye tracking, con el fin de determinar si las inserciones publicitarias tuvieron una buena visibilidad para el espectador, ya que este es el primer paso para lograr el recuerdo de la marca. También para medir el reconocimiento del consumidor sobre el producto anunciado. Tras el visionado se pidió a los participantes del estudio que completasen un formulario de tipo test y preguntas abiertas con el fin de identificar si reconocían Estrella Galicia y la recordaban entre los restantes productos y las otras marcas que también aparecían en los vídeos.

Para conocer la opinión que el espectador tenía de Estrella Galicia antes y después del visionado de los contenidos publicitarios, se les instó a hacer una valoración en Escala de Likert dos veces. También se les preguntó si la presencia de la marca les había resultado molesta y en qué medida, como forma de evaluar el rechazo sobre cada una de las dos modalidades publicitarias.

Finalmente el estudio se realizó en un contexto de exposición "on air" ya los participantes vieron el contenido publicitario en el mismo medio para el que fue concebido y se compararon productos creados para un mismo soporte (en este caso la televisión convencional) evitando así los sesgos diferenciadores. 


\section{Hipótesis de partida}

Para el estudio se proponen las siguientes hipótesis:

H1. El product placement favorece la visibilidad de la marca en mayor medida que el branded content. Para comprobar esta hipótesis se utilizará el estudio con eye tracking de los contenidos publicitarios.

$\mathrm{H} 2$. El product placement genera un mayor nivel de recuerdo en la mente del consumidor que el branded content. Para comprobar la hipótesis se someterá a los sujetos del estudio a un cuestionario de tipo test más preguntas abiertas, de recuerdo de marcas.

H3. El sentimiento positivo generado hacia la marca será mayor entre los sujetos que han consumido branded content que entre los que han visto product placement. Para ello se medirá en Escala de Likert la valoración que los usuarios hacen de la marca antes y después del visionado del contenido.

\section{Resultados de la investigación}

Tras el estudio realizado entre los dos grupos se extrajeron los siguientes resultados:

\subsection{En lo relativo a la visibilidad del producto}

En general, el contenido de branded de Estrella Galicia suele tener una mayor cantidad de primeros planos que en el caso del product placement, donde las ubicaciones son mayoritariamente pasivas y se encuentran de forma más habitual al fondo de los decorados. Este hecho determinó la visibilidad del producto en las distintas modalidades y afectó por lo tanto a los resultados, haciendo más notoria la presencia de la marca en el vídeo de branded content.

Por esta razón, el análisis comparativo de los resultados del eye tracking se hizo entre planos y ubicaciones de la marca similares en cuanto a cuadrante de pantalla y posición en primer término. De esta forma los resultados fueron comparables y sirvieron para confirmar que la visibilidad del producto como parte del attrezzo, aunque este se encuentre en un primer plano, es inferior que si forma parte activa del contenido de ocio. No importa por lo tanto la ubicación del producto, sino el hecho de que la acción principal se desarrolle sobre el mismo.

Esto contradice por lo tanto la hipótesis H1 (El product placement tiene una mayor visibilidad de la marca que el branded content) No solo fue más rápida la visualización del branded content, sino que el promedio de duración de la fijación de la mirada fue mayor que para el product placement (Fig. 1). Al interactuar más sobre el producto resulta más sencillo y natural que la mirada se centre más en el punto de pantalla en el que se encuentra ubicada la marca. 
Tabla. 1. Resultados de visualización de Estrella Galicia en product placement y branded content. Fuente: Elaboración propia.

\begin{tabular}{|c|c|c|}
\hline & $\begin{array}{c}\text { Tiempo hasta la } \\
\text { primera fijación }\end{array}$ & $\begin{array}{c}\text { Promedio de tiempo } \\
\text { de la fijación (en } \\
\text { segundos) }\end{array}$ \\
\hline Branded content & $0,81^{\prime \prime}$ & $0.48 \%$ \\
\hline $\begin{array}{c}\text { Product } \\
\text { placement }\end{array}$ & $1^{\prime} 47^{\prime \prime}$ & $0.45^{\prime}$ \\
\hline
\end{tabular}

Comparando los resultados de visibilidad en la zona de ubicación del producto con respecto de otras partes de pantalla, los resultados también fueron poco positivos para la publicidad insertada a modo de product placement (solo un 1,13\% de visibilidad en el área en la que se emplazaba Estrella Galicia). Para el branded content esa visibilidad fue en cambio de un 51\%. En ocasiones la zona en la que se ubicaba la marca era más visible que la zona en la que se desarrollaba la conversación entre el entrevistador y el invitado del programa (Fig. 1).

Fig. 1. Áreas de interés comparativas entre acción principal y producto en las modalidades de product placement y branded content.

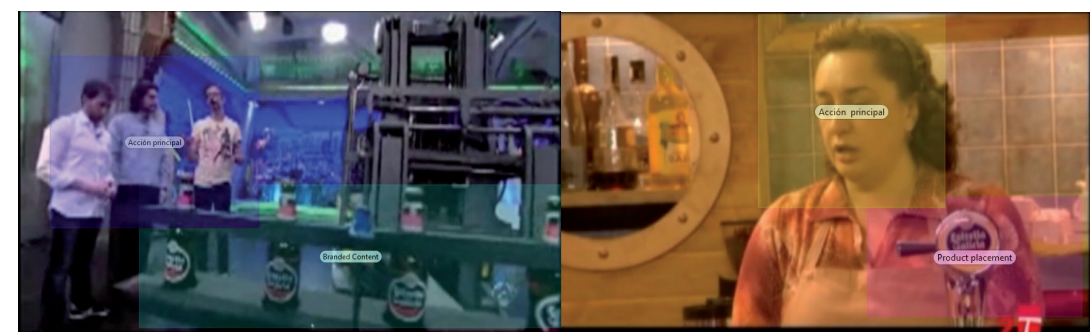

\subsection{En lo relativo al recuerdo de la marca anunciada}

En cuanto al análisis del recuerdo del producto, cabe destacar que Estrella Galicia fue la marca más recordada en los dos casos. Entre el grupo que observaba el product placement los usuarios la mencionaron en primer lugar (13 de los 15 usuarios del test), y todos los participantes dijeron haberla visto. En el caso del branded content los usuarios recordaron la marca de cervezas que aparecía en el programa. Por lo tanto, y a la vista de los resultados, ni se confirma ni se desmiente la hipótesis H2 de este estudio (El product placement genera un mayor nivel de recuerdo en la mente del consumidor que el branded content). La elevada presencia de la marca en las dos tipologías publicitarias hizo por lo tanto que la notoriedad de la marca fuese alta. Sin embargo, hay que destacar el hecho de que todos los participantes del estudio eran gallegos y consumidores de Estrella Galicia, lo que favoreció el índice de recuerdo ya que existía una predisposición positiva hacia la marca bastante alta (3,9 sobre 5, de media global). 


\subsection{En lo relativo a la percepción emotiva sobre la marca}

Por último, los resultados relativos a la valoración de marcas indicaron que los que vieron el contenido de product placement mejoraron su predisposición afectiva hacia Estrella Galicia ligeramente por encima de los que solo vieron el branded content.

Al grupo que vio el vídeo del programa El Hormiguero (branded content) se le pidió antes de empezar la prueba una valoración en escala de Likert de varias marcas de cerveza (Estrella Damm, Estrella Galicia, Voll Damm y Mahou). El objetivo era comprobar si existían cambios de valoración perceptiva sobre el anunciante que era objeto de estudio utilizando a la vez las otras marcas como elemento de despiste.

Los resultados indicaron una buena predisposición de partida hacia Estrella Galicia, ya que fue de todas la que contó con mejores valoraciones (Tabla 2).

Tabla 2. Valoración de Estrella Galicia previa y posterior al visionado del branded content. Fuente: Elaboración propia.

\begin{tabular}{|l|c|c|}
\hline & $\begin{array}{c}\text { Valoración antes del } \\
\text { visionado }\end{array}$ & $\begin{array}{c}\text { Valoración } \\
\text { posterior al } \\
\text { visionado }\end{array}$ \\
\hline Estrella Damm & 1,3 & $/$ \\
\hline Estrella Galicia & 4,1 & 4,4 \\
\hline Voll Damm & 1,4 & $/$ \\
\hline Mahou & 1,2 & $/$ \\
\hline
\end{tabular}

Sin embargo, la mejora en la percepción final para los participantes que vieron el branded content fue solo de 0,3 puntos. Entre los usuarios que hicieron el estudio sobre el contenido de product placement la valoración inicial de Estrella Galicia resultó ser inferior a la alcanzada entre los participantes del grupo branded content. En este caso a los usuarios se les pidió también que valorasen las otras marcas que habían ubicado sus productos en la serie (Tabla 3).

Tabla 3. Valoración de Estrella Galicia previa y posterior al visionado del product placement. Fuente: Elaboración propia.

\begin{tabular}{|l|c|c|}
\hline & $\begin{array}{c}\text { Valoración antes } \\
\text { del visionado }\end{array}$ & $\begin{array}{c}\text { Valoración } \\
\text { posterior al } \\
\text { visionado }\end{array}$ \\
\hline Estrella Galicia & 3,7 & 4,1 \\
\hline Abril & 1,2 & $/$ \\
\hline $\begin{array}{l}\text { La Voz de } \\
\text { Galicia }\end{array}$ & 3,2 & $/$ \\
\hline $\begin{array}{l}\text { Harley } \\
\text { Davidson }\end{array}$ & 3,4 & $/$ \\
\hline
\end{tabular}


Los resultados de la tabla 3 indican que tras el visionado del producto audiovisual la mejora de la percepción fue ligeramente superior en valores ( 0,4 puntos) que la que hubo en el grupo de los que visionaron el branded content. Se desmiente por lo tanto la hipótesis H3 (El sentimiento positivo generado hacia la marca será mayor entre los sujetos que han consumido branded content que en los que han visto product placement).

\section{Conclusiones}

De los resultados del estudio se concluye por lo tanto que, en líneas generales, el branded content alcanza una mayor visibilidad y recuerdo de marca que el product placement. El hecho de que el producto figure en pantalla como sujeto activo del contenido audiovisual hace, ya no solo que exista un mayor número de primeros planos sobre la marca, sino que resulte más natural al espectador dirigir la mirada hacia esa zona de la pantalla y que por lo tanto sea más consciente de la presencia del anunciante.

Sin embargo, esa interacción con el producto en la modalidad del product placement resultó ser menos eficiente. La visibilidad de la marca va a depender de la relevancia y el interés de las acciones principal y secundaria que se realicen en pantalla. Generalmente el consumo del producto se lleva a cambo como segunda acción (conducir un coche, abrir una bolsa de patatas fritas, servirse un vaso de leche, por ejemplo), al tiempo que los actores hacen otras cosas que llaman más la atención del espectador. Esto hace que el la atención se centre más en los gestos o la cara de los protagonistas que en la marca que pueda estar manipulando. Este factor afecta a la visibilidad que pueda tener el producto anunciado y, por lo tanto, a la efectividad que pueda tener la inserción publicitaria. Los resultados de visibilidad son peores en general porque las marcas anunciadas con product placement suelen hacerlo de forma pasiva, ubicando el producto en el decorado, casi siempre en el fondo. Además, lo habitual es que el escenario sea el soporte publicitario de más de una marca, con lo que la visibilidad y la eficiencia también se reducen por exceso de "ruido visual".

Eso sí, el número de impactos acostumbra a ser bastante mayor que en el caso del branded content, donde en ocasiones la marca ni siquiera está visible más que de forma verbal. Si el número de impactos aumenta, también lo hace la visibilidad, con lo que la marca resulta más notoria, aunque pueda precisamente saturar al público.

Por ello, para mejorar la eficacia del product placement es imprescindible mejorar la visibilidad de la marca, colocando los objetos en primer término y de forma activa, siendo parte protagonista de los guiones para que pueda ser reconocida de la manera más natural posible. Todo ello, lógicamente sin molestar o saturar al público.

El branded content puede incorporar mecánicas mucho más participativas y por ello se activa mejor el recuerdo de las marcas anunciantes entre los espectadores. Pese a ello, el producto publicitario no ha de ser en ningún caso molesto ni invasivo o la respuesta será contraproducente entre los consumidores, tal y como sucede en ocasiones con el product placement.

Resultan, por otro lado, sorprendentes los resultados del estudio relativos a la percepción y valoración de marca en cada una de las dos tipologías publicitarias. Dado que el branded content suele utilizar técnicas menos invasivas para llegar al 
consumidor, la percepción y reconocimiento sobre los productos que se anuncian utilizando esta modalidad debería ser menor. El product placement interrumpe el momento de ocio del consumidor y no es un contenido buscado, por lo que suele provocar rechazo. Sin embargo, a la vista de los resultados, el sentimiento de marca no empeora tras la exposición a la publicidad en ninguna de las dos modalidades analizadas e incluso resulta significativo el sentimiento positivo tras la exposición al product placement. Aunque ello no implique que dicha valoración sea más alta que tras la exposición al branded content, la mejora de la percepción sobre la marca anunciante fue mayor.

A raíz de estos resultados se puede considerar que las dos modalidades publicitarias analizadas mejoran el engagement sobre las marcas, creando valores y emociones que pueden ser positivas en torno a ellas, incrementando así la notoriedad del anunciante.

Sin embargo, cabe decir que para este estudio se utilizó una marca con un fuerte valor identitario y una imagen construida en torno a un valor sentimental bastante positivo entre el público general, tal y como demostraron los resultados de valoración en Escala de Likert al principio del estudio.

Por ello para comprobar la validez de los resultados acerca de la eficacia emotiva de cada una de las modalidades publicitarias analizadas sería necesario realizar el estudio de la valoración y el sentimiento medido en grupos de usuarios que no conozcan la marca que será objeto de estudio. De este modo se podría evitar cualquier tipo de predisposición afectiva previa que pueda afectar a los resultados y por lo tanto, medir de forma más objetiva cada una de las técnicas publicitarias. Igualmente, sería de interés ampliar el estudio a otros soportes digitales como móviles o tabletas, ya que en gran medida suelen formar parte de las estrategias de branded content de muchas marcas.

\section{Referencias bibliográficas}

Aguado, G. G. (2008) Branded content más allá del product placement en la televisión digital: Advertainment y licensing, en Revista del CES Felipe II (8) 5, http://www. cesfelipesegundo.com/revista/articulos2008/Art\%EDculo\%20Guadalupe\%20Aguado_ corregido.pdf Web visitada en diciembre 2015.

Babin, L. \& Carder, T. S. (1996) Viewers' recognition of brands placed within a film, en International Journal of Advertising 15 (2), 140-151.

Balasubramanian, S. K.; Karrh, J. A. \& Patwardhan, H. (2006) Audience Response to Product Placements: An Integrative Framework and Future Research Agenda, en Journal of Advertising 35 (3).

Baños, M. \& Rodríguez, T. (2004) Cuando la marca, marca la diferencia, en Enlaces 2, 1-9. a. (2012) Imagen de marca y product placement, Madrid, ESIC.

Caro, A. (2013) Del spot al branded content: lo audiovisual al servicio de las marcas, en Conferencia magistral. Congreso Internacional de Comunicación Facetas 4. Universidad Autónoma de Baja California. Ensenada, 5.11.2013. En: http://www.academia. edu/5035067/2013_-_Del_spot_al_branded_content._Lo_audiovisual_al_servicio_de_ las_marcas. Web visitada en julio 2016.

Cowley, E. \& Barron, C. (2008) When Product Placement Goes Wrong, en Journal of Advertising 37 (1), 89-98. 
Del Pino \& Olivares (2006) Brand placement y advertainment: integración y fusión entre la ficción audiovisual y las marcas, en Zer 22, 341-467.

Del Pino, C. \& Reinares, R. (2013) Evaluación y eficacia del branded content. Un estudio empírico, en Questiones publicitarias: revista internacional de comunicación y publicidad 18, 160-177.

Fernández, J. M., Reyes, I., Rocatagliata, E., Ruiz, E., Vargas, P. \& Pérez, J. P., (2012) Evolución del product placement en los films de James Bond: cincuenta años al servicio secreto de la publicidad, en Razón y Palabra 78, noviembre 2011 - enero 2012.

Gambetti, R. \& Graffigna, G. (2010) The concept of engagement. A systematic analysis of the ongoing marketing debate, en International Journal of Market Research 52 (6).

Gould, S.; Gupta, P. \& Grabner-Kräuter, S. (2000) Product placements in movies: a crosscultural analisys of Austrian, French and American consumers' attitudes toward this emerging, international promotional medium, en Journal of Advertising, 29 (4), 41-58.

Gupta, P. \& Gould, S. (1997) Consumers perceptions of the ethics and acceptability of product placement in movies: product category and individual differences, en Journal of Current Issues and Research Advertising 19 (1), 37-50.

Hollebeek, L. (2011) Exploring customer brand engagement: definition and themes, en Journal of Strategic Marketing 19 (7), 555-573. DOI: 10.1080/0965254X.2011.599493

Hudson, S. \& Hudson, D. (2006) Branded Entertainment: A New Advertising Technique or Product Placement in Disguise?, en Journal of Marketing Management 22:5-6, 489-504. DOI: $10.1362 / 026725706777978703$

KARrh, J. A. (1998): «Brand Placement: A Review.», Journal of Current Issues \& Research in Advertising, 20:2, pp. 31-49, DOI: 10.1080/10641734.1998.10505081

López, B. (2007) Publicidad emocional. Estrategias creativas, Madrid, ESIC.

Martínez, E., Montaner, T. \& Pina, J. M. (2005) Propuesta de medición de la imagen de marca. Un análisis aplicado a las extensiones de marca, en RAE: Revista Asturiana de Economía 33, 89-112.

Méndiz, A. (2007) Nuevas formas publicitarias. Patrocinio, Product Placement, Publicidad en Internet, Málaga, Universidad de Málaga.

Pergelova, A., Prior, D. \& Rialp, J. (2010) Assessing Advertising Efficiency, en Journal of Advertising 39 (3).

Regueira, J. (2012) El contenido como herramienta eficaz de comunicación de marca. Análisis teórico y empírico. Tesis Doctoral. Universidad Rey Juan Carlos. Departamento de economía de la empresa. Defendida en enero de 2012. En: https://eciencia.urjc.es/ handle/10115/11415 Web visitada en julio 2016.

Reijmersdal, E.A. (2009) Brand Placement Prominence: Good for Memory! Bad for Attitudes?, en Journal of Advertising Research 49 (2), 151-153. DOI: 10.2501/S0021849909090199

Rosique, G. (2010) El papel del telespectador en los medios audiovisuales. De homospectador a homo-civis, en Icono 14 (15), 147-163.

Verhellen, Y., Dens, N. \& De Pelsmacker, P. (2014) Context Matters: Modeling the impact of Context Perceptions on the Effectiveness of Brand Placement, en Advances in Advertising Research V. European Advertising Academy 5, 143-157.

Weller, R., Sibley, S. \& Neuhaus, C. (2015) Experimental Results Concerning the Affect of the Female Model in Television Commercials on Product and Brand Recall, en Proceedings of the 1982 Academy of Marketing Science (AMS) Annual Conference, 468-472. Springer.

Williams, K., Petrosky, A., Hernandez, E., \& Page, R. (2011) Product placement effectiveness: revisited and renewed, en Journal of Management and Marketing Research 7(1), 1-24. 
